Rev. Elev. Méd. vét. Pays trop., 1972, 25 (1): 15-19

\title{
Les mycobactéries atypiques d'origine animale étudiées à Dakar de 1966 à 1970
}

\author{
par J. CHAMBRON $(*)$, H. SARRAT (**) et Mme M. CASTETS $\left({ }^{* * *}\right)$
}

\section{RESUME}

\begin{abstract}
A la suite de la mise en évidence chez l'enfant à Dakar de syndromes pulmonaires dus à une mycobactérie atypique, les auteurs mènent de 1966 à 1970 une enquête systématique chez divers animaux domestiques ou sauvages, ou produits d'origine animale, dans le but de rechercher un éventuel réservoir animal de germes pour ces mycobactéries atypiques à pouvoir potentiel pour l'homme. Ils isolent 10 souches à partir de 213 prélèvements. La plupart sont des saprophytes.

L'importance respective de ces germes dans la pathologie animale ouest-africaine, plus spécialement par rapport à la tuberculose vraie, leur pouvoir pathogène pour l'homme, sont discutés.
\end{abstract}

Le terme de tuberculose est actuellement réservé aux infections dues à Mycobacterium tuberculosis et Mycobacterium bovis, et celui de mycobactériose sert à désigner les infections provoquées par des mycobactéries différentes de ces deux types bacillaires et couramment appelées mycobactéries atypiques. Celles-ci, au même titre que les premières, rentrent également dans le cadre des anthropozoonoses. En effet, de très nombreux travaux et observations démontrent la fréquence d'isolement, chez les animaux ou dans les aliments d'origine animale, de ces mycobactéries atypiques à pouvoir pathogène potentiel ou réel pour l'homme.

En zone intertropicale, et plus spécialement en Afrique de l'Ouest, avant 1966, les recherches dans ce domaine sont rares ou même inexistantes, l'accent étant mis en priorité sur le

(*) Service de Bactériologie, Laboratoire national de l'Elevage du Sénégal, B.P. 2057, Dakar.

(**) Service de Bactérılogie, Institut Pasteur de Dakar, B.P. 220.

(**) Service de Bactériologie-Virologie de la Faculté mixte de Médecine et de Pharmacie de J'Uriversité de Dakar. rôle et l'importance de la tuberculose, singulièrement de la tuberculose bovine.

Mais, à partir de 1966, des études plus approfondies entreprises dans différents laboratoires de cette partie de l'Afrique amènent à reconnaître, à côté de l'infection tuberculeuse, la présence chez l'homme de mycobactéries atypiques, dont la pathogénicité a quelquefois pu être établie avec certitude. Ces cas, rencontrês en clinique hospitalière courante, nous ont amenés tout naturellement à entreprendre des enquêtes systématiques dans l'environnement et chez plusieurs espèces animales, dans le but de mieux connaître l'épidémiologie de ces mycobactéries dans nos régions, et de mettre éventuellement un réservoir de germes animal en évidence.

Les résultats de ces enquêtes, ainsi que les quelques commentaires qu'ils suscitent, constituent l'objet de la présente note.

\section{ORIGINE DES PRELEVEMENTS - TECHNIQUES}

Nous ne dirons rien de spécial au sujet des techniques de culture et d'identification des 
souches de mycobactéries, qui sont celles classiquement utilisées en la matière.

Les souches obtenues à partir des milans, des vautours et des tourterelles lors des enquêtes épidémiologiques systématiques sont isolées à partir de broyats " aveugles " de foie et de rate; celles des porcs, à partir de broyats des ganglions mésentériques. Aucun de ces animaux ne présente de lésions anatomo-pathologiques pouvant évoquer la tuberculose.

Ces prélèvements animaux sont décontaminés à l'aide du bromure de cétylpyridinium, dont nous avons souligné l'efficacité par ailleurs (3).

En ce qui concerne les laits, sources fréquentes de contamination par des mycobactéries atypiques en Europe, il s'agit également d'analyses " aveugles" pratiquées sur des boues industrielles résultant de la centrifugation de laits de grands mélanges dans une usine de la région de Saint-Louis. Il convient de souligner la difficulté d'isolement de mycobactéries à partir de ces produits généralement très souillés et qui nécessitent une méthode de décontamination à la fois très efficace et très sélective. Nous avons adopté la technique préconisée par TACQUET et ses collaborateurs, basée sur l'action successive et ménagée du lauryl sulfate de sodium alcalin et de l'acide oxalique (11).

\section{RESULTATS}

Les mycobactéries que nous avons étudiées pendant la période de temps envisagée peuvent être rangées dans deux catégories distinctes:

- Une première catégorie regroupe les souches d'origine animale isolées à Dakar à l'occa- sion de diagnostics courants ou adressées par d'autres territoires de l'Ouest africain (HauteVolta, Mali et Niger) pour identification complète (tableau I).

- La seconde catégorie comprend les souches que nous avons isolées et étudiées à Dakar à l'occasion de nos enquêtes épidémiologiques systématiques chez différentes espèces animales ou à partir de produits d'origine animale (tableau II).

Toutes les mycobactéries atypiques identifiées ont été classées dans l'un des quatre grands groupes classiques définis par RUNYON en 1959 selon la rapidité de leur croissance et leur aptitude à produire des pigments à la lumière du jour ou à l'obscurité :

\section{- Groupe 1 :}

Mycobactéries photochromogènes, à croissance lente, et dont les colonies non pigmentées à l'obscurité se pigmentent après une courte exposition à la lumière.

\section{- Groupe 2 :}

Mycobactéries scotochromogènes, à croissance lente, et dont les colonies se pigmentent spontanément à l'obscurité.

\section{- Groupe 3:}

Mycobactéries non chromogènes, dont les colonies, à croissance lente, ne se pigmentent habituellement ni à la lumière ni à l'obscurité.

\section{- Groupe 4 :}

Mycobactéries à croissance rapide, les colonies se développant en 3 à 4 jours.

Les résultats obtenus sont résumés dans les deux tableaux suivants :

TABIEAU N*II

Mycobactéries atypiques isolêes à Dakar à l'occasion d'enquêtes êpidémiologiques systëmatiques.

\begin{tabular}{|c|c|c|c|c|c|}
\hline \multirow{2}{*}{ Espềces animales } & \multirow{2}{*}{$\begin{array}{l}\text { Noụbre de } \\
\text { prélèvements } \\
\text { êtudiês }\end{array}$} & \multicolumn{3}{|c|}{ Mycobactéries atypiques isolées } & \multirow{2}{*}{ Tatal } \\
\hline & & Groupe II & Groupe III & Groupe IV & \\
\hline Milans et vautours & 50 & 2 & & & 2 \\
\hline Tourterelles & 45 & & & & 0 \\
\hline Porcs & 83 & 3 & & & 3 \\
\hline Lait & 35 & & 1 & 4 & 5 \\
\hline Total & 213 & 5 & 1 & 4 & 10 \\
\hline
\end{tabular}


TABLEAU $N^{\circ} \mathrm{I}$

Mycobactéries atypiques reçues de divers Etats d'Afrique de 1'Ouest pour identification, ou isolêes à Dakar.

\begin{tabular}{|l|c|c|c|c|c|}
\hline & Groupe I & Groupe II & Groupe III & Groupe IV & Tota1 \\
\cline { 2 - 6 } Bovins & 1 & 2 & 1 & & 4 \\
Porcins & & & 1 & 1 & 1 \\
Oigeaux & & & 2 & 1 & 1 \\
\hline Total & 1 & 2 & & & 6 \\
\hline
\end{tabular}

\section{DISCUSSION}

Le nombre des souches isolées étant assez réduit, ces résultats apparaissent bien décevants et n'incitent guère à de larges commentaires.

Deux aspects du problème de ces mycobactéries atypiques d'origine animale méritent cependant d'être discutés, celui de l'importance de ces germes dans la pathologie animale en Afrique de l'Ouest, et celui du danger de contamination qu'ils représentent pour l'homme.

\section{Importance des mycobactéries atypiques} dans la pathologie animale ouest africaine, et plus spécialement par rapport

à la tuberculose vraie

Il est sans doute utile de rappeler ici que la présence d'une mycobactérie atypique dans un prélèvement, qu'il soit d'ailleurs d'origine animale ou humaine, ne suffit pas à démontrer la pathogénicité réelle du bacille. Les chiffres rapportés dans le tableau I ne préjugent donc en rien d'une infection mycobactérienne authentique. Cependant des mycobactéries atypiques sont retrouvées de plus en plus souvent chez des animaux, dans des affections rappelant le processus tuberculeux. Parmi divers articles consacrés à ce sujet, qu'il nous soit permis de citer celui de OUDAR et collab. (7) qui font une revue intéressante de la question.

Par ailleurs, les différences de répartition géographique de la tuberculose animale, bovine et porcine principalement, rendent difficile une estimation de l'incidence réelle des mycobactérioses par rapport à l'infection tuberculeuse vraie. En effet, dans l'Ouest africain, si la Haute-Volta et le Mali sont des Etats où l'infection tuberculeuse est la plus répandue, le Niger reste peu touché et, au Sénégal, elle est pratiquement inconnue. L'importance des mycobactérioses animales par rapport à la tuberculose différera donc sensiblement d'un Etat à l'autre.
Enfin, les chiffres exposés dans le tableau I ne concernent que des souches de mycobactéries isolées lors d'examens de routine (cas clinique, mise en évidence de lésions suspectes lors d'une inspection sanitaire à l'abattoir, ...) et adressées à Dakar aux fins d'identification; nous ne pouvons par conséquent faire état du nombre total de prélèvements examinés par rapport au nombre de ceux reconnus authentiquement tuberculeux. On peut noter cependant que lors d'une enquête réalisée à Bobo-Dioulasso en Haute-Volta de 1965 à 1968, GIDEL et collab. (6) isolent à partir de carcasses saisies pour tuberculose 13 mycobactéries atypiques représentant 5,2 p. 100 du total des cultures positives obtenues à partir de 639 prélèvements soumis à examen. Dans ce travail comme dans celui d'ALBERT et collab. (1), les auteurs précisent que ces souches d'origine animale sont isolées à partir de lésions anatomo-pathologiques bien caractérisées qu'il est difficile de différencier microscopiquement des lésions dues à des mycobactéries typiques, $M$. bovis par exemple. En ce qui nous concerne, de 1966 à 1970 , soit la période de temps couverte par notre étude, le pourcentage de mycobactéries atypiques d'origine ouest-africaine identifiées à Dakar, par rapport aux bacilles tuberculeux type humain ou bovin, est de 6,4 p. 100 .

Ces données sont donc insuffisantes pour juger de l'incidence réelle des mycobactérioses animales en pathologie vétérinaire; mais la présence de tels germes chez l'animal amène à considérer la possibilité d'une contamination humaine éventuelle, deuxième point de notre discussion.

\section{Pouvoir pathogène pour l'homme des mycobactéries atypiques}

La plupart des mycobactéries atypiques que l'on rencontre dans la nature sont en général considérées comme des saprophytes. C'est 
notamment le cas des bacilles des groupes II et IV. Il n'en est pas de même pour les mycobactéries des groupes I et III dont le caractère pathogène pour l'homme ou pour l'animal est plus souvent reconnu et vérifié [OUDAR et collab. (7) déjà cités].

De nombreux travaux ont en effet montré la présence fréquente de mycobactéries du groupe aviaire chez des oiseaux, des porcs, des bovins ou dans des boues de centrifugation industrielle de grands mélanges de laits de vache, posant ainsi la question de l'existence d'un réservoir animal de germes pour ce groupe bacillaire (5).

Le pouvoir pathogène de ces mycobactéries a été d'autre part largement démontré chez l'homme en pays tempérés (4). Au Sénégal, récemment, l'un de nous a pu constater l'existence chez le jeune enfant d'un syndrome pulmonaire à forme de primo-infection, dont la responsabilité a pu être attribuée à des mycobactéries du groupe aviaire (8). Des enquêtes tuberculiniques effectuées notamment en zone rurale ont d'ailleurs confirmé la réalité de ce fait en révélant un pourcentage relativement important de sujets réagissant aux sensitines spécifiques de ce groupe (2).

Il était donc intéressant de rechercher chez l'animal et d'une manière plus systématique la présence de telles mycobactéries, en s'adressant plus spécialement aux espèces reconnues comme pouvant être une source d'infection.

Les résultats de nos enquêtes (voir tableau 2) sont malheureusement peu démonstratifs, et seule la présence d'une mycobactérie du groupe III de Runyon dans un culot de centrifugation de lait doit être retenue, sans pouvoir affirmer pour autant que ce germe a une origine mammaire certaine. Ces résultats, somme toute négatifs, corroborent ceux que nous avions enregistrés au cours d'une enquête tuberculinique parallèle humaine et animale effectuée en zone rurale (9) : les animaux testés (bovins et volailles) n'avaient montré aucune trace aliergique d'infection à bacilles aviaires, alors qu'un nombre assez élevé d'enfants du même village avaient réagi aux sensitines spécifiques de ce groupe.

\section{CONCLUSION}

Au terme d'une étude qu'il nous a paru utile de présenter, nous pouvons retenir que les infections vraies à mycobactéries atypiques constituent chez l'animal une entité pathologique encore difficile à estimer, en raison peutêtre du faible intérêt qu'une telle recherche a suscité jusqu'à présent; cette étude a cependant permis de reconnaître que les animaux, en hébergeant des mycobactéries atypiques, sont susceptibles d'être une source de contamination humaine mais ne représentent probablement pas un réservoir de germes important. II paraît donc raisonnable d'adopter l'opinion de SCHAEFER (10) qui évoquait la forte probabilité d'une contamination humaine et animale à partir d'une même source qui pourrait être le sol.

Cette hypothèse constitue sans nul doute une base de recherche qui, outre son originalité en zone intertropicale, permettrait de mieux connaître l'épidémiologie des mycobactérioses, affections encore mal connues dans nos régions.

\section{SUMMARY}

\section{Study in Dakar of animal atypical mycobacteria (1966-1970)}

The part played by atypic mycobacteria in pulmonary syndromes in children has been demonstrated in Dakar 3 years ago. In the present paper, the authors publish the results of a systematic survey carried out between 1966 and 1970 on various domestic and wild animals and animal products. This survey was aimed to detect a possible animal reservoir for these atypic mycobacteria presenting a pathogenic incidence to man. 10 strains were isolated from 213 samples. Most of them were saprophyte.

The importance of these germs in the west african animal pathogenesis, particularly in regard of true $T B$, and their potential danger to man are discussed. 


\section{RESUMEN}

\section{Las micobacterias atipicas de origen animal estudiadas en Dakar} de 1966 a 1970

Luego del evidenciar en el niño de sindromes pulmonares causados por una micobacteria atipica, los autores efectuaron, de 1966 a 1970, una encuesta sistemática en varios animales domésticos o salvajes, o en productos de origen animal, para buscar un reservorio animal eventual de germenes para estas micobacterias atipicas teniendo un poder patógeno en el hombre. Aislaron 10 cepas a partir de 213 muestras. La mayor parte de dichas son saprofitas.

Se discuten la importancia respectiva de estos germenes en la patologia animal del oeste de Africa, más especialmente en relación con la tuberculosis verdadera, y su poder patógeno en el hombre.

\section{BIBLIOGRAPHIE}

1. ALBERT (J. P.), GIDEL (R.), LEFEVRE (M.), RETIF (M.), DJOKOUI et CAUSSE (G.), CHAMBON (L.) et SARRAT (H.), Mycobactéries d'origine animale isolées au Centre Muraz de 1965 à 1967, Méd. Afr. noire, 1969, 16 (4) : 335-336.

2. ARNAUD (P.), SARRAT (H.) et SATGE (P.), Etude de l'allergie due aux mycobactéries atypiques chez l'enfant sénégalais, Bull. Soc. méd. Afr. noire, 1070,15 (1): 42-55.

3. CHAMBRON (J.) et SARRAT (H.), Résultats d'une étude comparative du lauryl-sulfate de sodium et du bromure de cétylpyridinium pour l'iso'ement de mycobactéries à partir de prélèvements animaux et humains, Rev. Elev Méd. vét. Pays trop., 1971, 24 (2) : 173-181.

4. GERBEAUX (J.) et collab., Table ronde sur les infections à mycobactéries atypiques chez l'enfant et chez l'adulte, Cahiers Coll. med., 1969, 10 (2): 119-143.

5. GERNEZ-RIEUX (Ch.), TACQUET (A.), DEVULDER (B.) et DEBRUYNE (J.), Les mycobactérioses humaines - aspects épidémiologiques, $\mathbf{X V I}^{\mathrm{e}}$ Congrès de la tuberculose, New York, 1969.
6. GIDEL (R.), ALBERT (J. P.), LEFEVRE (M.), MENARD (M.) et RETIF (M.), Les mycobactéries d'origine animale isolées au Centre Muraz de 1965 à 1968 - Techniques d'isolement et d'identification - Résultats, Rev. Elev. Méd. vét. Pays trop., 1969, 22 (4) : 495-508.

7. OUDAR (J.), JOUBERT (J.), VALLIER (J.), CAILLERE (F.) et GORET (P.), Les mycobactéries atypiques chez les animaux. Leur éventuelle transmission à l'homme, Rev. Path. comp., sept. 1966: 447-491.

8. SARRAT (H.), SENGHOR (G.), TRENOU (R.) et CONGY (F.), Place des mycobactéries atypiques du groupe III de Runyon dans la pathologie d'un service de Pédiatrie à Dakar, Bull. Soc. méd. Afr. noire, 1968,13 (2) : 273-286.

9. SARRAT (H.) et CHAMBRON (J.), Résultats d'une enquête tuberculinique humaine et animale effectuée en zone rurale au Sénégal, Bull. Soc. Path. exot., 1969, 62 (6) : 992-1000.

10. SCHAEFER (W. B.), Amer. Rev, resp. Dis., 1968, 97: 18-23.

11. TACQUET (A.), TISON (F.), DEVULDER (B.) et ROOS (P.), Ann. Inst. Pasteur, Lille, 1966, 17 : $161-172$ et $173-180$. 